\title{
Etude des composants de la flaveur du yoghourt
}

\author{
par \\ M. GROUX \\ Département de Recherche et Développement \\ Société d'Assistance Technique pour Produits Nestlé, S.A. \\ Case Postale 1009, CH-1001 Lausanne \\ Directeur du Département R \& D : Professeur Louis REY
}

\section{INTRODUCTION}

Il existe une très abondante littérature au sujet de l'arôme et de la flaveur du yoghourt. Certains auteurs attribuent une importance à la caséolyse aromatisante (Carini, 1969). Selon cet auteur, la protéolyse est due surtout au Str. thermophilus et apparaît donc au début de la fermentation.

D'autres auteurs (Miller et Kandler, 1967) ont mis en évidence que la protéolyse est intense selon la souche utilisée et que certains acides aminés - en particulier l'acide glutamique, la proline, la leucine, la valine et la sérine - sont produits en grandes quantités.

L'importance organoleptique des arômes volatils ne fait plus de doute. On admet généralement que le composant le plus important est l'acétaldéhyde. Les autres composés - diacétyle et acétoïne sont considérés comme des substances d'influence mineure. Certains auteurs (Lindsay, 1966) attribuent un faible pouvoir aromatisant à l'acétoïne par exemple.

L'étude présentée permet de préciser quelques points qui n'ont pas retenu toute l'attention des chercheurs laitiers. Certaines considérations sont fondées sur des résultats obtenus avec du yoghourt séché.

\section{MATERIEL ET METHODES}

\section{1) Souches bactériennes}

Les souches microbiennes lyophilisées proviennent des Laboratoires de Biologie Appliquée (25, av. Michelet (93) Saint-Ouen, France). Elles ont été entretenues sur lait écrémé autoclavé contenant 1 p. 1000 d'extrait de levures (Difco). 


\section{2) Préparation de yoghourt frais et de yoghourt séché}

Le yoghourt a été fabriqué soit en laboratoire, soit à l'échelle pilote à partir de lait écrémé ou demi-écrémé upérisé. Dans le cas du yoghourt séché, le lait frais était additionné de saccharose et de gélatine alimentaire dans des proportions sur matières solides totales, respectivement, de 23.4 et 3.6 p. 100 . Le lait inoculé avec 3 p. 100 de cultures de yoghourt était acidifié en 2 h 30 à 3 h à 43 à $44^{\circ} \mathrm{C}$ jusqu'à un $\mathrm{pH}$ entre 4.7 et 4.6 puis refroidi. Le $\mathrm{pH}$ après refroidissement se situait généralement entre 4.5 et 4.4 .

Selon le cas, le yoghourt a été concentré sous vide puis pulvérisé dans un sécheur du type "spray".

\section{3) Dosages des arômes volatils}

L'acétaldéhyde, le diacétyle et l'acétoïne ont été dosés par les méthodes utilisées déjà par V. Bottazzi et M. Vescovo (1969).

\section{4) Dosages des composants non volatils}

Les acides aminés libres ont été dosés par la méthode chromatographique à l'Amino Acid Analyser Beckman après ultrafiltration sur membrane Sartorius (nitrate de cellulose No SM121.36) non perméable aux molécules de plus de 50 A.

L'ammoniac a été dosé à partir d'un distillat à la vapeur d'eau d'une suspension de yoghourt neutralisée par $\mathrm{K}_{2} \mathrm{HPO}_{4} \mathrm{M}$ à $\mathrm{pH}: 8.0$ par titration avec $\mathrm{H}_{2} \mathrm{SO}_{4} 0.02 \mathrm{~N}$.

\section{5) Evaluations organoleptiques}

Celles-ci ont été effectuées par un groupe de 11 personnes spécialement formées et l'évaluation exécutée selon le principe du classement dit de "Ranking ". Chaque membre du panel recevait des échantillons de yoghourt présentés dans des tasses émaillées noir intérieurement.

La salle de dégustation était éclairée en lumière rouge afin d'uniformiser les couleurs des échantillons. Les dégustateurs étaient priés de classer l'intensité et la fraîcheur de la flaveur du yoghourt dans l'ordre suivant :

- goût très fin, typique de yoghourt,

- diminution du goût typique,

- atypique,

- très plat.

\section{RESULTATS}

\section{1) La production d'acides aminés libres}

Nous avons comparé les teneurs en acides aminés libres d'un même lait écrémé upérisé et acidifié par : 
- voie chimique (addition de $\mathrm{HCl}$ O.IN),

- voie biologique (cultures de yoghourt des établissements BIA).

Les deux laits acidifiés jusqu'à pH 4.6 ont été ensuite centrifugés à $2500 \mathrm{~g}$ pendant $20 \mathrm{mn}$. Les surnageants ont été congelés rapidement et lyophilisés.

Pour les deux types de lait acidifié, les pourcentages de $\mathrm{N}$ total sur la matière sèche des surnageants lyophilisés étaient les suivants :

- voie chimique : 0.82 p. 100 ,

— voie biologique : 0.90 p. 100.

Rapportées à la quantité du $\mathrm{N}$ total du lait écrémé de départ, les quantités du $\mathrm{N}$ sur les surnageants étaient dans les deux cas de 11.5 et 11.7 p. 100 respectivement.

Les quantités en acides aminés libres dosés dans l'ultrafiltrat étaient de :

— voie chimique : $80.5 \mathrm{mg}$ par $100 \mathrm{~g}$ de filtrat $\mathrm{sec}$,

— voie biologique : $213.5 \mathrm{mg}$ par $100 \mathrm{~g}$ de filtrat sec,

TABLEAU 1. - Dosage des acides aminés libres dans le yoghourt (en $\mathrm{mg} 100 \mathrm{~g}$ de filtrat sec)

\begin{tabular}{|c|c|c|}
\hline Acide aminé & Acidification chimique & Acidification biologique \\
\hline Lysine & 8.3 & 7.2 \\
\hline Histidine & - & 17.0 \\
\hline Arginine & - & - \\
\hline Ac. aspartique & 5.1 & 7.5 \\
\hline Thréonine & 2.4 & 2.4 \\
\hline Sérine & 6.3 & 15.0 \\
\hline Ac. glutamique & 41.5 & 61.0 \\
\hline Proline & - & 70.5 \\
\hline Glycine & 12.8 & 2.8 \\
\hline Alanine & 4.1 & 11.7 \\
\hline Cystine & - & - \\
\hline Valine & - & 3.1 \\
\hline Méthionine & - & 0.8 \\
\hline Isoleucine & - & 1.5 \\
\hline Leucine & - & 5.8 \\
\hline Tyrosine & - & 6.1 \\
\hline Phénylalanine & - & 1.1 \\
\hline Tryptophane & pas dosé & pas dosé \\
\hline Total & 80.5 & 213.5 \\
\hline $\mathrm{N}^{-\mathrm{NH}_{3}}$ (dosé séparément) & 19 & 108 \\
\hline
\end{tabular}

(L'absence de chiffre signifie l'absence d'acide aminé libre ou en quantité non mesurable dans les conditions d'analyse). 
non compris les teneurs en tryptophane qui n'a pas été dosé. Les teneurs respectives en les différents acides aminés sont les suivantes : (voir tab. 1).

Ces résultats montrent les différences qualitatives et quantitatives entre les deux acidifications du lait. On remarque que le filtrat du yoghourt contient tous les acides aminés à l'exclusion de l'arginine et de la cystine. Généralement, les quantités de chaque acide aminé sont supérieures dans le filtrat du yoghourt. Il est intéressant de noter que le filtrat du yoghourt contient moins de glycine libre. Ceci est aussi valable dans une mesure moindre pour la lysine. Les quantités de thréonine libre des deux laits sont les mêmes.

La production de $\mathrm{NH}_{3}$ par les bactéries du yoghourt a quintuplé la quantité originelle présente dans le lait.

\section{2) La production microbienne d'arômes volatils et son incidence sur la flaveur}

On a effectué deux préparations de yoghourt en poudre à partir de lait demi-écrémé additionné de saccharose et de gélatine. Le lait upérisé puis ensemencé et acidifié a été concentré sous vide et séché.

Des échantillons ont été prélevés aux stades suivants :

- après acidification et refroidissement,

- après concentration sous vide,

- après séchage.

Les dosages d'arômes ont donné les résultats suivants :

TABLEAU 2

Pertes d'arômes volatils lors de la concentration sous vide et du séchage spray $\mathrm{du}$ yoghourt (concentration en $\mathrm{mg}$ par $\mathrm{kg}$ de matières solides)

\begin{tabular}{|c|c|c|c|}
\hline & Acétaldéhyde & Diacétyle & Acétoïne \\
\hline $\begin{array}{l}\text { Essai } n^{\circ} 1 \\
\text { après acidification } \\
\text { après concentration } \\
\text { après séchage }\end{array}$ & $\begin{array}{r}110 \\
18 \\
7\end{array}$ & $\begin{array}{r}9 \\
6 \\
<5\end{array}$ & $\begin{array}{l}74 \\
31 \\
17\end{array}$ \\
\hline $\begin{array}{l}\text { Essai } n^{\circ} 2 \\
\text { après acidification } \\
\text { après concentration } \\
\text { après séchage }\end{array}$ & $\begin{array}{r}92 \\
13 \\
<5\end{array}$ & $\begin{array}{r}20 \\
10 \\
<5\end{array}$ & $\begin{array}{r}200 \\
110 \\
28\end{array}$ \\
\hline
\end{tabular}

On remarquera que les teneurs en diacétyle et acétoïne des yoghourts frais sont très différentes. 


\section{3) Résultats de dégustation des échantillons de yoghourt}

Les échantillons de yoghourt concentré et séché ont été dilués à une teneur en matière sèche identique avant d'être présentés au groupe de dégustateurs. Ceux-ci n'avaient pas à tenir compte des différences de couleur ou de texture mais étaient priés de se prononcer uniquement sur l'intensité et la fraîcheur de la flaveur de yoghourt.

\section{TABLEAU 3}

Intensité et fraîcheur de la flaveur de différents yoghourts (réponses en p. 100)

\begin{tabular}{|c|c|c|c|c|}
\hline & $\begin{array}{l}\text { Goût très fin, } \\
\text { typique du } \\
\text { yoghourt }\end{array}$ & $\begin{array}{l}\text { Diminution } \\
\text { du goût } \\
\text { typique }\end{array}$ & Atypique & $\begin{array}{l}\text { Très } \\
\text { plat }\end{array}$ \\
\hline $\begin{array}{l}\text { Essai } n^{\circ} 1 \\
\quad \text { après acidification } \\
\text { après concentration } \\
\text { après séchage }\end{array}$ & $\begin{array}{r}100 \text { p. } 100 \\
18 \text { p. } 100 \\
27 \text { p. } 100\end{array}$ & $\begin{array}{l}54 \text { p. } 100 \\
18 \text { p. } 100\end{array}$ & $\begin{array}{l}18 \text { p. } 100 \\
36 \text { p. } 100\end{array}$ & $\begin{array}{l}10 \text { p. } 100 \\
18 \text { p. } 100\end{array}$ \\
\hline $\begin{array}{l}\text { Essai } n^{\circ} 2 \\
\text { après acidification } \\
\text { après concentration } \\
\text { après séchage }\end{array}$ & $\begin{array}{r}100 \text { p. } 100 \\
18 \text { p. } 100 \\
10 \text { p. } 100\end{array}$ & $\begin{array}{l}82 \text { p. } 100 \\
18 \text { p. } 100\end{array}$ & $\frac{\overline{-}}{27 \text { p. } 100}$ & $\frac{\overline{-}}{45 \text { p. } 100}$ \\
\hline
\end{tabular}

On remarquera que les résultats des dégustations ont dans les grandes lignes respecté la prévision logique. D'autre part, les résultats confirment ceux du dosage des composants volatils. La disparité des réponses des dégustations augmente quand les teneurs en arômes volatils sont faibles. Fait intéressant, la concentration sous vide n'est pas jugée très négativement, la plupart des dégustateurs estimant que le produit n'est pas encore atypique. Certains dégustateurs jugent même le produit concentré comme peu différent du produit initial et les classent ensemble.

Le séchage par contre est jugé comme étant très défavorable en conséquence des pertes d'arômes mais à des degrés divers.

\section{DISCUSSION}

La protéolyse du lait par les ferments du yoghourt est peu spécifique puisque tous les acides aminés importants - à l'exception de l'arginine et de la cystine - apparaissent sous forme libre dans le 
yoghourt. Les quantités de chaque acide aminé libre formé ne sont pas en relation avec l'abondance de ces acides aminés dans les protéines du lait.

Pour certains acides aminés, les quantités trouvées dans le yoghourt sont inférieures à celles du lait de départ (glycine, lysine). Il semble donc qu'une partie des acides aminés libres du lait soit consommée au cours de l'acidification par les cultures du yoghourt. Il n'est pas impossible que ces acides aminés libres soient des facteurs de croissance importants pour les ferments du yoghourt.

La quantité d'acides aminés libres trouvée dans le yoghourt n'est donc que le résultat net de la fermentation mais elle ne donne pas une image complète des acides aminés libres réellement secrétés par les bactéries ou libérés des protéines par protéolyse.

Les bactéries du yoghourt sont aussi de fortes productrices de $\mathrm{NH}_{3}$. Dans le yoghourt frais, il est peu probable que $\mathrm{NH}_{3}$ soit un métabolite bactérien car la fermentation a été arrêtée au moment où les micro-organismes se trouvaient encore dans une phase de forte multiplication (phase logarithmique).

Il est raisonnable de penser que $\mathrm{NH}_{3}$ peut provenir d'une désamination qui, sans qu'il y ait de preuves absolues à disposition, pourrait avoir lieu au niveau de certains acides aminés libres. Remarquons d'emblée que ceci ne permet pas d'expliquer l'absence d'arginine libre. Si cet acide aminé est désaminable selon le tableau de G. Michal (1972), selon Bergey (1957) et M. Sharpe et T. Fryer (1968), le Str. thermophilus et le $L b$ bulgaricus ne sont pas producteurs d' $\mathrm{NH}_{3}$ à partir de l'arginine. Le cas de la cystine est différent : la désamination de cet acide aminé peut donner l'acide pyruvique. La cystine libre pourrait dès lors être un substrat énergétique d'appoint d'un ou des micro-organisme (s) du yoghourt.

Un fait intéressant concerne la proline libre qui est présente en grandes quantités dans le yoghourt. Il est tentant de relier l'abondance en cet acide aminé libre au fait qu'il n'est probablement pas désaminable enzymatiquement.

La thèse de la désamination des acides aminés libres est attractive à plus d'un titre. En effet, elle permettrait peut-être d'expliquer au moins en partie l'origine de certains composants de la flaveur du yoghourt. Par exemple l'acide propionique pourrait provenir d'une désamination de la $\beta$-alanine. La thréonine pourrait selon un même schéma être désaminée puis décarboxylée pour donner l'acétone.

L'importance de la protéolyse pour la flaveur du yoghourt prendrait dès lors une signification nouvelle. Les faibles quantités d'acides aminés libres présents dans le yoghourt n'ont probablement pas d'importance per se. Par contre, en temps que précurseurs d'arômes ces acides aminés jouent peut-être un plus grand rôle.

En ce qui concerne les arômes volatils que nous avons dosés, il semble que l'on puisse attribuer une importance primordiale à 
l'acétaldéhyde. Néanmoins, le rôle du diacétyle et de l'acétoïne n'est pas à négliger. En effet, les dégustateurs ont préféré de manière quasi unanime les échantillons du deuxième essai en lui attribuant une finesse de flaveur plus grande que pour le premier essai. Or ce qui distingue le deuxième essai du premier ce sont les teneurs en diacétyle et acétoïne. Il se pourrait que ces deux composants renforcent le caractère de fraîcheur conféré par l'acétaldéhyde. De plus, dans les cas où les teneurs en acétaldéhyde sont faibles (par ex. après concentration) les quantités de diacétyle encore assez importantes pourraient maintenir un certain degré de finesse de la flaveur. Ceci pourrait aussi expliquer les jugements de classement divergents de certains dégustateurs.

\section{R é s u m é}

La flaveur du yoghourt est attribuée surtout à la présence de composés volatils à seuils de perception assez bas. Si l'acétaldéhyde est le composé dont la présence en quantités importantes est caractéristique du yoghourt frais, il est montré dans quelle mesure le diacétyle et l'acétoïne peuvent se substituer en partie à lui dans les cas où il fait défaut.

Le présent travail apporte une contribution à l'étude de l'importance de la protéolyse pour la flaveur du yoghourt. Les résultats font penser que les acides aminés libres produits lors de la fermentation du lait par les bactéries du yoghourt pourraient être des précurseurs de certains composés aromatiques qui contribuent à la finesse de la flaveur du yoghourt.

\section{S u $\mathbf{m} \mathbf{m}$ a r y}

\section{Study on the components of yoghurt flavor}

The typical yoghurt flavor is generally attributed to volatile components of low theshold values.

The presence of relatively high amounts of acetaldehyde is characteristic of the aroma of fresh yoghurt.

Our results indicate that when acetaldehyde is present in very small quantities, diacetyl and even acetoin can partly substitute it. Thus the typical yoghurt flavor is still maintained.

The present study also shows the importance of the enzymatic proteolysis for the development of yoghurt flavor. In addition, our work suggest that the free aminoacids resulting from the proteolysis could be precursors of some other aromatic components which contribute to the delicacy of yoghurt flavor. 


\section{Remerciements}

Nous remercions tout particulièrement Mlle E. Bujard pour ies dosages d'acides aminés libres et Mlle N. Daget qui a pris en charge les tests d'évaluation organoleptique. Les dosages d'arômes volatils ont été exécutés avec compétence par Mme F. Truffer que nous remercions tout spécialement.

\section{Références bibliographiques}

Bergey's Manual of Determinative Bacteriology, 7th Ed., Williams and Wilkins, 1957.

Miller (J.) und Kandler (O.) (1967). - Milchw., 22 (8), 469-480.

SHARPe (M.) and FrYer (T.) (1968). - Identification Methods for Microbiologists. Ac. Press.

Bottazzi (V.) and Vescovo (M.) (1969). - Neth. Milk Dairy, J. 23, 71-78.

CARINI (S.) et al. (1969). - Scienza Tec. latt.-casear., 20 (5), 289-304.

Michal (G.). - Biochemical Pathways, Ed. 1972, Boehringer Mannheim GmBH, Germany. 